\title{
Summability of Independent Random Variables
}

\section{WILLIAM E. PRUITT ${ }^{1}$}

\author{
Communicated by M. Rosenblatt
}

1. Introduction. Let $\left\{X_{k}\right\}$ be a sequence of independent, identically distributed random variables with $E\left|X_{k}\right|<\infty$ and $E X_{k}=\mu$. Let $A=\left(a_{n k}\right)$ be a Toeplitz summation matrix, i.e.

$$
\begin{aligned}
\lim _{n \rightarrow \infty} a_{n k} & =0 \text { for every } k, \\
\lim _{n \rightarrow \infty} \sum_{k=1}^{\infty} a_{n k} & =1, \text { and } \\
\sum_{k=1}^{\infty}\left|a_{n k}\right| & \leqq M \text { for all } n .
\end{aligned}
$$

(Condition (1.2) may be omitted when $\mu=0$.) Since

$$
E \sum_{k=1}^{\infty}\left|a_{n k} X_{k}\right|=E\left|X_{k}\right| \sum_{k=1}^{\infty}\left|a_{n k}\right| \leqq M E\left|X_{k}\right|
$$

the series $\sum_{k} a_{n k} X_{k}$ converges absolutely with probability one. The purpose of this paper is to study the convergence properties of the sequence $Y_{n}=$ $\sum_{k} a_{n k} X_{k}$ as $n \rightarrow \infty$.

In the next section it is shown that $Y_{n} \rightarrow \mu$ in probability if and only if $\max _{k}\left|a_{n k}\right| \rightarrow 0$. (The trivial case when $X_{k}$ is almost surely equal to $\mu$ must be omitted here.) This condition is not enough, however, to guarantee almost sure (a.s.) convergence. The main result here is

Theorem 2. If $\max _{k}\left|a_{n k}\right|=O\left(n^{-\gamma}\right), \gamma>0$, then $E\left|X_{k}\right|^{1+1 / \gamma}<\infty$ implies that $Y_{n} \rightarrow \mu$ a.s.

The proof is in Section 3. Examples are given in Section 4 to show that Theorem 2 is sharp in the sense that for every $\gamma>0$, there exists a matrix $A$ with $\max _{k}\left|a_{n k}\right|=O\left(n^{-\gamma}\right)$ such that $Y_{n} \rightarrow \mu$ a.s. implies $E\left|X_{k}\right|^{1+1 / \gamma}<\infty$.

1 This work was partially supported by the Air Force Office of Scientific Research. 
Theorem 2 was suggested by the theorem of Katz [3] which states that for $t \geqq 1, E\left|X_{k}\right|^{t}<\infty$ and $E X_{k}=\mu$ if and only if

$$
\sum_{n=1}^{\infty} n^{t-2} P\left[\left|\sum_{k=1}^{n} X_{k}-n \mu\right|>n \epsilon\right]<\infty
$$

for every $\epsilon>0$. To illustrate the connection, we consider the case $t=2$. The matrix $A$ with elements

$$
a_{n k}=\left\{\begin{array}{l}
1 / n \text { for } j_{n-1}<k \leqq j_{n}, \\
0 \text { otherwise }
\end{array}\right.
$$

where $j_{n}=n(n+1) / 2$, is a Toeplitz matrix with $\max _{k}\left|a_{n k}\right|=n^{-1}$. Furthermore $Y_{n} \rightarrow \mu$ a.s. if and only if the series in (1.4) converges (for $t=2$ ) by the Borel Zero-One Criterion [4, p. 228]. This is the example which shows that the theorem is sharp when $\gamma=1$. The proof of the theorem uses methods of Erdös [1] which were extended by Katz in [3].

The situation when the matrix $A$ is determined by a single sequence $\left\{w_{k}\right\}$ of positive numbers by the relation

$$
a_{n k}=\left\{\begin{array}{cl}
w_{k} / W_{n} & \text { if } 1 \leqq k \leqq n, \\
0 & \text { if } \quad k>n,
\end{array}\right.
$$

where $W_{n}=\sum_{k=1}^{n} w_{k}$ is treated in [2]. If the $A$ matrix has this extra structure, then the results of [2] are much sharper than those given here.

2. Convergence in probability. Let $F$ be the common distribution function of the $X_{k}$ 's and $X$ a random variable having this distribution. It is also convenient to adopt the convention that when $a_{n k}=0,\left|a_{n k}\right|^{-1}=+\infty$. The case when $X$ is equal to $\mu$ a.s. must clearly be omitted in order to obtain the necessity stated in the following theorem.

Theorem 1. A necessary and sufficient condition that $Y_{n} \rightarrow \mu$ in probability is that $\max _{k}\left|a_{n k}\right| \rightarrow 0$ as $n \rightarrow \infty$.

Proof. The proof of sufficiency is very similar to the corresponding argument in [2], but it will be given here for the sake of completeness. First, we have that

$$
\lim _{T \rightarrow \infty} T P[|X| \geqq T]=0
$$

since $E|X|<\infty$. Let $X_{n k}$ be $a_{n k} X_{k}$ truncated at one, and $Z_{n}=\sum_{k} X_{n k}$. Now for all $n$ sufficiently large, since $\max _{k}\left|a_{n k}\right| \rightarrow 0$, it follows from (2.1) that

$P\left[Z_{n} \neq Y_{n}\right] \leqq \sum_{k} P\left[X_{n k} \neq a_{n k} X_{k}\right]=\sum_{k} P\left[|X| \geqq \frac{1}{\left|a_{n k}\right|}\right] \leqq \epsilon \sum_{k}\left|a_{n k}\right| \leqq \epsilon M$.

It will therefore suffice to show that $Z_{n} \rightarrow \mu$ in probability. Note that

$$
E Z_{n}-\mu=\sum_{k} a_{n k}\left[\int_{|x|<\left|a_{n k}\right|-1} x d F-\mu\right]+\mu\left[\sum_{k} a_{n k}-1\right] \rightarrow 0 .
$$


Since

$$
\frac{1}{T} \int_{|x|<T} x^{2} d F=\frac{1}{T}\left\{-T^{2} P[|X| \geqq T]+2 \int_{0}^{T} x P[|X| \geqq x] d x\right\} \rightarrow 0,
$$

it follows that for all $n$ sufficiently large

$$
\sum_{k} \operatorname{Var} X_{n k} \leqq \sum_{k}\left|a_{n k}\right|^{2} \int_{|x|<\left|a_{n k}\right|^{-1}} x^{2} d F \leqq \epsilon \sum_{k}\left|a_{n k}\right| \leqq \epsilon M .
$$

But $E\left(\sum_{k}\left|X_{n k}\right|\right)^{2}$ is easily seen to be finite so that $\operatorname{Var} Z_{n}=\sum_{k} \operatorname{Var} X_{n k}$ which tends to zero by (2.2). An application of Chebyshev's inequality completes the proof of sufficiency.

For the necessity, let $U_{k}=X_{k}-\mu, T_{n}=\sum_{k} a_{n k} U_{k}$ so that $T_{n} \rightarrow 0$ in probability and hence in law. Let $g(u)=E e^{i u U_{k}}$ be the characteristic function of $U_{k}$. We have that $\prod_{k=1}^{\infty} g\left(a_{n k} u\right) \rightarrow 1$ as $n \rightarrow \infty$. But

$$
\left|\prod_{k=1}^{\infty} g\left(a_{n k} u\right)\right| \leqq\left|g\left(a_{n m} u\right)\right| \leqq 1
$$

for any $m$, so that for any sequence $k_{n}$,

$$
\left|g\left(a_{n k_{n}} u\right)\right| \rightarrow 1 \text {. }
$$

Since $U_{k}$ is non-degenerate, there is a $u_{0}$ such that $|g(u)|<1$ for $0<|u|<u_{0}$ [4, p. 202]. Letting $u=u_{0} / 2 M$, it follows that

$$
\left|a_{n k_{n}} u\right| \leqq M u=u_{0} / 2
$$

and then

$$
a_{n k_{n}} u \rightarrow 0
$$

by (2.3). Choosing $k_{n}$ to satisfy

$$
\left|a_{n k_{n}}\right|=\max _{k}\left|a_{n k}\right|
$$

completes the proof.

3. Almost sure convergence. The proof of Theorem 2 will be an easy consequence of the following three lemmas.

Lemma 1. If $E|X|^{1+1 / \gamma}<\infty$ and $\max _{k}\left|a_{n k}\right| \leqq B n^{-\gamma}$, then for every $\epsilon>0$, $\sum_{n} P\left[\left|a_{n k} X_{k}\right| \geqq \epsilon\right.$ for some $\left.k\right]<\infty$.

Proof. It suffices to consider $B=1$ and $\epsilon=1$ for both the matrix $A$ and the random variables $X_{k}$ may be multiplied by a positive constant if necessary. (Assumption (1.2) is not used in this proof.) Let

$$
N_{n}(x)=\sum_{\left\{k:\left|a_{n k}\right|^{-1} \geqq x\right\}}\left|a_{n k}\right| \text {. }
$$

Notice that $N_{n}(x)=0$ for $x<n^{\gamma}$ and $\int_{0}^{\infty} d N_{n}(x)=\sum_{k}\left|a_{n k}\right| \leqq M$. If $G(x)=$ 
$P[|X| \geqq x], \lim T G(T)=0$ as $T \rightarrow \infty$ since $E|X|<\infty$, and thus

$$
\begin{aligned}
\sum_{k} P\left[\left|a_{n k} X_{k}\right| \geqq 1\right] & =\sum_{k} G\left(\left|a_{n k}\right|^{-1}\right)=\int_{0}^{\infty} x G(x) d N_{n}(x) \\
& =\lim _{r \rightarrow \infty} T G(T) N_{n}(T)-\int_{0}^{\infty} N_{n}\left(x^{-}\right) d(x G(x)) \\
& \leqq M \int_{n \gamma}^{\infty} d|x G(x)| .
\end{aligned}
$$

To estimate the last integral, observe that for $z<y$,

$$
y G(y)-z G(z)=(y-z) G(z)+y(G(y)-G(z))
$$

with the first term increasing and the second decreasing as a function of $y$. Therefore

$$
\int_{z}^{y} d|x G(x)| \leqq(y-z) G(z)+y(G(z)-G(y))
$$

so that

$$
\begin{aligned}
\int_{n^{\gamma}}^{\infty} d|x G(x)| & =\sum_{j=n}^{\infty} \int_{j^{\gamma}}^{(i+1)^{\gamma}} d|x G(x)| \\
& \leqq \sum_{j=n}^{\infty}\left[(j+1)^{\gamma}-j^{\gamma}\right] G\left(j^{\gamma}\right)+\sum_{j=n}^{\infty}(j+1)^{\gamma}\left[G\left(j^{\gamma}\right)-G\left((j+1)^{\gamma}\right)\right] .
\end{aligned}
$$

Summing the first of the final series by parts and using the existence of $E|X|$, we see that it is dominated by the second series, and thus

$$
\int_{n \gamma}^{\infty} d|x G(x)| \leqq 2 \sum_{i=n}^{\infty}(j+1)^{\gamma}\left[G\left(j^{\gamma}\right)-G\left((j+1)^{\gamma}\right)\right] .
$$

Finally, by (3.1) and (3.2),

$$
\begin{aligned}
& \sum_{n=1}^{\infty} P\left[\left|a_{n k} X_{k}\right| \geqq 1 \text { for some } k\right] \leqq \sum_{n=1}^{\infty} \sum_{k=1}^{\infty} P\left[\left|a_{n k} X_{k}\right| \geqq 1\right] \\
& \leqq 2 M \sum_{n=1}^{\infty} \sum_{j=n}^{\infty}(j+1)^{\gamma}\left[G\left(j^{\gamma}\right)-G\left((j+1)^{\gamma}\right)\right] \\
&=2 M \sum_{j=1}^{\infty} j(j+1)^{\gamma}\left[G\left(j^{\gamma}\right)-G\left((j+1)^{\gamma}\right)\right] \\
& \leqq 2^{\gamma+1} M \int|x|^{1+1 / \gamma} d F(x)<\infty .
\end{aligned}
$$

Lemma 2. If $E|X|^{1+1 / \gamma}<\infty$ and $\max _{k}\left|a_{n k}\right| \leqq B n^{-\gamma}$, then for $\alpha<\gamma / 2(\gamma+1)$, $\sum_{n} P\left[\left|a_{n k} X_{k}\right| \geqq n^{-\alpha}\right.$ for at least two values of $\left.k\right]<\infty$.

Proof. By the Markov inequality,

$$
P\left[\left|a_{n k} X_{k}\right| \geqq n^{-\alpha}\right] \leqq\left|a_{n k}\right|^{1+1 / \gamma} E|X|^{1+1 / \gamma} n^{\alpha(1+1 / \gamma)},
$$


so that

$$
\begin{aligned}
P\left[\left|a_{n k} X_{k}\right|\right. & \left.\geqq n^{-\alpha} \text { for at least two } k\right] \\
& \leqq \sum_{j \neq k} P\left[\left|a_{n j} X_{j}\right| \geqq n^{-\alpha},\left|a_{n k} X_{k}\right| \geqq n^{-\alpha}\right] \\
& \leqq\left(E|X|^{1+1 / \gamma}\right)^{2} n^{2 \alpha(1+1 / \gamma)} \sum_{j \neq k}\left|a_{n j}\right|^{1+1 / \gamma}\left|a_{n k}\right|^{1+1 / \gamma} \\
& \leqq\left(E|X|^{1+1 / \gamma}\right)^{2} B^{2 / \gamma} M^{2} n^{2[-1+\alpha(1+1 / \gamma)]},
\end{aligned}
$$

and the final estimate will converge when summed on $n$ provided that $\alpha<\gamma / 2(\gamma+1)$.

Lemma 3. If $\mu=0, E|X|^{1+1 / \gamma}<\infty$, and $\max _{k}\left|a_{n k}\right| \leqq B n^{-\gamma}$, then for every $\epsilon>0, \sum_{n} P\left[\left|\sum_{k}^{\prime} a_{n k} X_{k}\right| \geqq \epsilon\right]<\infty$, where

$$
\sum_{k}^{\prime} a_{n k} X_{k}=\sum_{\left\{k:\left|a_{n k} X_{k}\right|<n-\alpha\right\}} a_{n k} X_{k}
$$

and $0<\alpha<\gamma$.

Proof. Let

$$
X_{n k}= \begin{cases}X_{k} & \text { if }\left|a_{n k} X_{k}\right|<n^{-\alpha} \\ 0 & \text { otherwise }\end{cases}
$$

and $\beta_{n k}=E X_{n k}$. If $a_{n k}=0$, then $\beta_{n k}=\mu=0$, while if $a_{n k} \neq 0$, then

$$
\left|\beta_{n k}\right|=\left|\mu-\int_{|x| \geqq n^{-\alpha}\left|a_{n k}\right|^{-1}} x d F\right| \leqq \int_{|x| \geqq n^{-\alpha_{B}-1} n}|x| d F .
$$

Therefore $\beta_{n k} \rightarrow 0$ uniformly in $k$ and $\sum_{k} a_{n k} \beta_{n k} \rightarrow 0$. Let $Z_{n k}=X_{n k}-\beta_{n k}$ so that $E Z_{n k}=0, E\left|Z_{n k}\right|^{1+1 / \gamma} \leqq c$ for some $c$, and $\left|a_{n k} Z_{n k}\right| \leqq 2 n^{-\alpha}$. Now

$$
\sum_{k}^{\prime} a_{n k} X_{k}=\sum_{k} a_{n k} X_{n k}=\sum_{k} a_{n k} Z_{n k}+\sum_{k} a_{n k} \beta_{n k}
$$

and so for $n$ sufficiently large

$$
\left[\left|\sum_{k}^{\prime} a_{n k} X_{k}\right| \geqq \epsilon\right] \subset\left[\left|\sum_{k} a_{n k} Z_{n k}\right| \geqq \epsilon / 2\right] .
$$

It will suffice, therefore, to show that

$$
\sum_{n} P\left[\left|\sum_{k} a_{n k} Z_{n k}\right| \geqq \epsilon\right]<\infty .
$$

Let $\nu$ be the least integer greater than $1 / \gamma$. The necessary estimate will be obtained by computing $E\left(\sum_{k} a_{n k} Z_{n k}\right)^{2 \nu}$ and using Markov's inequality. It is readily seen that $E\left(\sum_{k}\left|a_{n k} Z_{n k}\right|\right)^{2 \nu}$ is finite so that

$$
E\left(\sum_{k} a_{n k} Z_{n k}\right)^{2 \nu}=\sum_{k_{1}}, \cdots, k_{2}, ~ E \prod_{i=1}^{2 v} a_{n k_{j}} Z_{n k_{i}}
$$

There is no contribution to the sum on the right so long as there is a $j$ with 
$k_{i} \neq k_{i}$ for all $i \neq j$ since the $Z_{n k}$ are independent and $E Z_{n k}=0$. The general term to be considered then will have

$$
\begin{aligned}
& q_{1} \text { of the } k^{\prime} \mathrm{s}=\xi_{1}, \cdots, q_{m} \text { of the } k^{\prime} \mathrm{s}=\xi_{m}, \\
& r_{1} \text { of the } k^{\prime} \mathrm{s}=\eta_{1}, \cdots, r_{p} \text { of the } k^{\prime} \mathrm{s}=\eta_{p},
\end{aligned}
$$

where $2 \leqq q_{i} \leqq 1+1 / \gamma, r_{i}>1+1 / \gamma$, and

$$
\sum_{i=1}^{m} q_{i}+\sum_{j=1}^{p} r_{i}=2 \nu .
$$

Then

$$
\begin{aligned}
& E \prod_{i=1}^{m}\left(a_{n \xi_{i}} Z_{n \xi_{i}}\right)^{a_{i}} \prod_{i=1}^{p}\left(a_{n \eta_{i}} Z_{n \eta_{j}}\right)^{r_{j}} \\
& \quad \leqq(1+c)^{\nu} \prod_{i=1}^{m}\left|a_{n \xi_{i}}\right|^{a_{i}} \prod_{i=1}^{p}\left|a_{n \eta_{j}}\right|^{1+1 / \gamma}\left(2 n^{-\alpha}\right)^{\left(r_{j}-1-1 / \gamma\right)} \\
& \leqq(1+c)^{\nu} \prod_{i=1}^{m}\left|a_{n \xi_{i}}\right| \prod_{j=1}^{p}\left|a_{n \eta_{i}}\right|\left(B n^{-\gamma}\right)^{\sum_{i=1}^{m}\left(a_{i}-1\right)+p / \gamma}\left(2 n^{-\alpha}\right)^{\sum_{i=1}^{p}\left(r_{i}-1-1 / \gamma\right)},
\end{aligned}
$$

where $c$ is the upper bound for $E\left|Z_{n k}\right|^{1+1 / \gamma}$ mentioned above. Now the power to which $n$ is raised is the negative of

$$
\gamma \sum_{i=1}^{m}\left(q_{i}-1\right)+p+\alpha \sum_{i=1}^{p}\left(r_{i}-1-1 / \gamma\right) .
$$

Now if $p$ is one (or larger),

$$
p+\alpha \sum_{i=1}^{p}\left(r_{i}-1-1 / \gamma\right) \geqq 1+\alpha(\nu-1 / \gamma),
$$

while if $p=0$,

$$
\gamma \sum_{i=1}^{m}\left(q_{i}-1\right)=\gamma(2 \nu-m) \geqq \gamma \nu=1+\gamma(\nu-1 / \gamma) \geqq 1+\alpha(\nu-1 / \gamma),
$$

the first inequality being a result of

$$
m \leqq \frac{1}{2} \sum_{i=1}^{m} q_{i}=\nu .
$$

Therefore the expectation in (3.4) is bounded by

$$
K_{1} \prod_{i=1}^{m}\left|a_{n \xi_{i}}\right| \prod_{i=1}^{p}\left|a_{n \eta_{i}}\right| n^{-1-\alpha(\nu-1 / \gamma)}
$$

and $K_{1}$ depends only on $c, \gamma$, and $B$. It follows that

$$
E\left(\sum_{k} a_{n k} Z_{n k}\right)^{2 \nu} \leqq K_{2} n^{-1-\alpha(\nu-1 / \gamma)}
$$


for some $K_{2}$ which may depend on $c, \gamma, B$, and $M$ but is independent of $n$. An application of the Markov inequality now yields (3.3).

Proof of Theorem 2. Observe that

$$
\sum_{k} a_{n k} X_{k}=\sum_{k} a_{n k}\left(X_{k}-\mu\right)+\mu \sum_{k} a_{n k}
$$

and the last term converges to $\mu$ by (1.2). Therefore, we may consider only the case $\mu=0$. By the Borel-Cantelli Lemma, it suffices to show that for every $\epsilon>0$,

$$
\sum_{n} P\left[\left|\sum_{k} a_{n k} X_{k}\right| \geqq \epsilon\right]<\infty
$$

But

$$
\begin{gathered}
{\left[\left|\sum_{k} a_{n k} X_{k}\right| \geqq \epsilon\right] \subset\left[\left|\sum_{k}^{\prime} a_{n k} X_{k}\right| \geqq \frac{\epsilon}{2}\right] \cup\left[\left|a_{n k} X_{k}\right| \geqq \frac{\epsilon}{2} \text { for some } k\right]} \\
\cup\left[\left|a_{n k} X_{k}\right| \geqq n^{-\alpha} \text { for at least two } k\right] .
\end{gathered}
$$

Now, if $0<\alpha<\gamma / 2(\gamma+1)$, then $\alpha<\gamma$ also and the series (3.5) converges as a consequence of the three lemmas.

4. Examples demonstrating the sharpness of theorem 2. These examples are similar to the one given in the introduction for the case $\gamma=1$. First, suppose that $0<\gamma<1$. Let $n_{i}=\min \left\{m: m^{\gamma} \geqq i\right\}$ for $i \geqq 0, j_{0}=0$, and for $n_{i-1}<n \leqq n_{i}$, let $j_{n}=n i-\sum_{m=1}^{i-1} n_{m}$. Then the matrix $A$ with elements

$$
a_{n k}=\left\{\begin{array}{l}
1 / i \text { if } n_{i-1}<n \leqq n_{i} \text { and } j_{n-1}<k \leqq j_{n} \\
0 \text { otherwise }
\end{array}\right.
$$

is a Toeplitz matrix with $\max _{k}\left|a_{n k}\right|=O\left(n^{-\gamma}\right)$ such that if $\sum_{k} a_{n k} X_{k} \rightarrow \mu$ a.s., then $E|X|^{1+1 / \gamma}<\infty$. This is seen by a straightforward application of Katz theorem.

For $\gamma>1$, define $j_{n}=\left[n^{\gamma}\right]+1$ and $k_{n}=\sum_{i=1}^{n} j_{i}$. The matrix $A$ with elements

$$
a_{n k}=\left\{\begin{array}{cl}
1 / j_{n} & \text { for } k_{n}<k \leqq k_{n+1} \\
0 & \text { otherwise }
\end{array}\right.
$$

is a Toeplitz matrix since $j_{n+1} / j_{n} \rightarrow 1$, and

$$
\max _{k}\left|a_{n k}\right|=\frac{1}{j_{n}} \leqq n^{-\gamma} \text {. }
$$

Assume that $\sum_{k} a_{n k} X_{k} \rightarrow \mu$ a.s. Given $\epsilon>0$ and the distribution function $F$, let $\rho_{n}$ be the integer $m$ which maximizes

$$
P\left[\left|\sum_{i=1}^{m} X_{i}-m \mu\right|>m \epsilon\right]
$$


in the range $j_{n} \leqq m<j_{n+1}$. Let $B$ be the matrix with elements

$$
b_{n k}=\left\{\begin{array}{cl}
1 / \rho_{n} & \text { if } k_{n}<k \leqq k_{n}+\rho_{n}, \\
0 & \text { otherwise. }
\end{array}\right.
$$

The first step is to show that $\sum_{k} b_{n k} X_{k} \rightarrow \mu$ a.s. This is a consequence of the assumption that $\sum_{k} a_{n k} X_{k} \rightarrow \mu$ a.s. together with an application of the classical strong law to the $X_{k}$ 's with indices such that $k_{n}+\rho_{n}<k \leqq k_{n+1}$. From this point on, the fact that $E|X|^{1+1 / \gamma}<\infty$ follows from Katz theorem very much as before.

\section{BIBLIOGRAPHY}

[1] Erdös, P. On a theorem of Hsu and Robbins, Ann. Math. Statist., 20 (1949) 286-291.

[2] Jamison B. Orey, S. \& Pruitr, W., Convergence of weighted averages of independent random variables, $Z$. Wahrscheinlichkeitstheorie und Verw. Gebiete., 4 (1965) 40-44.

[3] Katz, M. L., The probability in the tail of a distribution, Ann. Math. Statist., 34 (1963) 312-318.

[4] Loฌ̀ve, M., Probability Theory, New York, Van Nostrand, 1955.

University of Minnesota 